\title{
Bibliothèques, conservatoires, observatoires
}

Nouveaux matériaux pour l'histoire de l'astronomie moderne : autour du landgraviat de Hessen-Butzbach, 1609-1630

\section{Pierre Jeandillou et Édouard Mehl}

\section{(2) OpenEdition}

1 Journals

Édition électronique

URL : http://journals.openedition.org/rbnu/608

DOI : $10.4000 /$ rbnu. 608

ISSN : 2679-6104

Éditeur

Bibliothèque nationale et universitaire de Strasbourg

\section{Édition imprimée}

Date de publication : 1 novembre 2018

Pagination : $62-71$

ISBN : 9782859230784

ISSN : 2109-2761

Référence électronique

Pierre Jeandillou et Édouard Mehl, « Bibliothèques, conservatoires, observatoires », La Revue de la BNU [En ligne], 18 | 2018, mis en ligne le 01 mai 2019, consulté le 10 décembre 2020. URL : http:// journals.openedition.org/rbnu/608; DOI : https://doi.org/10.4000/rbnu.608

\section{(c) (1) (-)}

La Revue de la BNU est mise à disposition selon les termes de la Licence Creative Commons Attribution - Pas d'Utilisation Commerciale - Partage dans les Mêmes Conditions 4.0 International. 


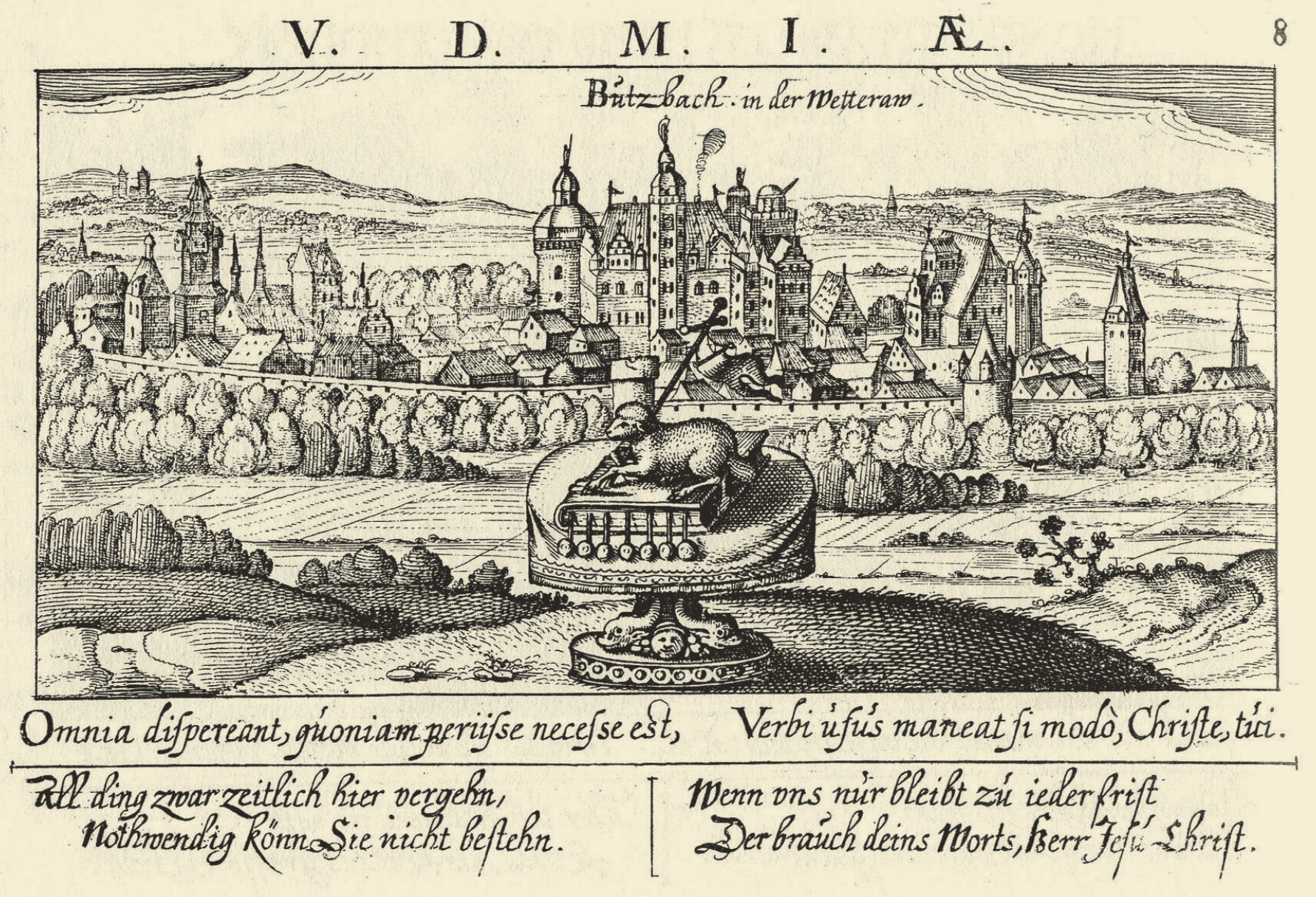

Vue de Butzbach in der Wetterau (1625). La tour d'angle (à droite) montre le tube optique braqué vers l'horizon (in Daniel Meisner, Eberhard Kieser, Thesaurus Philopoliticus oder Politisches Schatzkästlein, Bd. 1, Buch 5, Nr. 8, fac-similé de l'édition de Frankfurt-am-Main, 1625-1626 et 1627-1631, Nördlingen, 1992). 


\title{
BIBLIOTHÈQUES, CONSERVATOIRES, OBSERVATOIRES
}

\author{
Nouveaux matériaux pour l'histoire \\ de l'astronomie moderne: autour du landgraviat \\ de Hessen-Butzbach, 1609-1630
}

PAR PIERRE JEANDILLOU ET ÉDOUARD MEHL

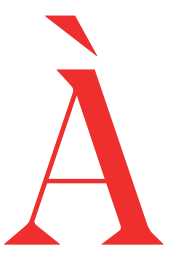

la mort du landgrave Philippe $\mathrm{I}^{\mathrm{er}}$ de Hesse « le Magnanime » (15041567), qui fut l'un des principaux soutiens politiques de Luther au temps de la Réforme, le vaste territoire sur lequel il régnait fut partagé entre ses quatre fils : la Hesse-Kassel échut à son premier fils Guillaume IV (1532-1592) ; la Hesse-Marbourg à son second fils Louis IV (1537-1604) ; la Hesse-Rheinfels à Philippe II (1541-1583), et la Hesse-Darmstadt à Georges I ${ }^{\text {er }}$ (1547-1596). À la mort de Louis IV, en 1604, celui-ci n'ayant pas d'héritier, la Hesse-Marbourg fut partagée entre ses neveux, et Marbourg elle-même fut rattachée à la Hesse-Kassel, où le fils et successeur de Guillaume IV, Moritz von Hessen-Kassel (1572-1632), y imposa le calvinisme, tandis que les parties échues aux landgraves de Darmstadt demeuraient dans la confession luthérienne.

Sous le regard attentif du roi de France Henri IV ${ }^{1}$, une longue rivalité politique et confessionnelle entre Kassel et Darmstadt, attisée par le conflit généralisé de la guerre de Trente Ans (1618-1648), structure ce paysage politique morcelé et passablement instable, qui favorise néanmoins l'autonomie temporaire des plus petites subdivisions, comme le landgraviat de Hessen-Butzbach, administré de manière indépendante par le landgrave Philipe III (1581-1643). En 1607 , les landgraves de Darmstadt et Butzbach fondent l'université luthérienne de Gießen, pour contrecarrer l'influence grandissante de Kassel et de la réforme calviniste imposée à l'université de Marbourg.

\section{_ La bibliothèque du " liber naturae "}

Dès ses années de formation, Philippe de Hessen-Butzbach manifeste un intérêt précoce pour les questions scientifiques. Le landgrave fait partie $\mathrm{du}$ très petit nombre de ceux qui ont eu le privilège d'avoir des contacts épistolaires directs à la fois avec l'« excellentissime et doctissime Signor Galileo Galilei » ${ }^{2}$ et, un peu plus tard, avec le mathématicien impérial Johannes Kepler. Dès son accession au pouvoir, en 1609 - quand commencent à circuler en Europe la lunette de Galilée et son Messager céleste annonçant en termes sobres et concis la découverte, sidérante et irrécusable, 


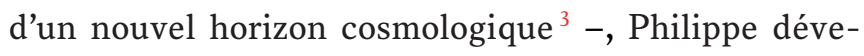
loppe la bibliothèque et fait faire des transformations dans le château pour y installer un observatoire astronomique (1625, voir ill. p. 62). L'inventaire de cette bibliothèque aujourd'hui dispersée est d'un intérêt majeur pour l'histoire des sciences à l'âge classique. Établi tout au long de la guerre de Trente Ans par le mathématicien Daniel Mögling (1596-1635), dont le landgrave s'est adjoint les services à partir de l'année 1621, l'inventaire comporte, outre les livres répertoriés selon leur discipline d'appartenance (arithmétique, géométrie, astronomie, astrologie, géographie, architecture, chimie, botanique...), deux inventaires complémentaires distincts : l'un concerne les maquettes et modèles réduits ( "Allerley Modelle», f. 13) ; l'autre, qui nous intéressera plus spécifiquement ici, concerne les instruments astronomiques d'observation et de mesure (« Allerley Instrumenta », ff. 14-17) ${ }^{4}$. L'observatoire de Butzbach fournit l'exemple paradigmatique d'une bibliothèque moderne, entièrement, exclusivement et presque religieusement consacrée au déchiffrement du «livre de la nature $»^{5}$.

Bien que l'activité scientifique du landgrave et de son ingénieur d'études aient contribué de manière décisive, au dire de Kepler lui-même, au progrès de la recherche scientifique dans la première moitié du $17^{\mathrm{e}}$ siècle, cet apport est resté jusqu'à aujourd'hui largement méconnu. Cet oubli a une double explication. D'une part, Philippe III n'ayant pas de successeur, Butzbach a perdu son indépendance politico-administrative après sa mort, en 1643. Une partie de la bibliothèque de la "Reißkammer " ${ }^{6}$ est revenue, via Marburg, à l'université de Gießen ; l'autre a disparu sans laisser de traces. Mais une autre raison, moins évidente, peut expliquer cette disparition. Elle tient à la biographie et à la personnalité de celui qui joue ici un rôle déterminant : Daniel Mögling. Avant de rentrer comme en religion au service du landgrave, celui-ci a commis, sous des pseudonymes variés, de nombreux opuscules apologétiques en faveur de la Fraternité de la RoseCroix $^{7}$. Il est également très lié à un mathématicien à la réputation sulfureuse, Johann Faulhaber (15801635), dont les vaticinations apocalyptiques défraient la chronique et lui valent même, au cours de l'année 1619 , un procès à Ulm, où il est sommé de justifier ses opinions hétérodoxes et de clarifier la teneur de ce que ses contemporains appellent, non sans ironie, sa « cabale log-arithmo-géo-mantique ${ }^{8}$. Daniel Mögling, protégé de Faulhaber, aurait pu être sérieusement inquiété s'il n'avait alors trouvé refuge à la cour de Butzbach et gardé l'anonymat dans les années qui suivirent. Kepler, qui le connaît assez pour lui confier la garde de sa fille Suzanne dans les années 1620, ne prononce jamais publiquement son nom, bien que Mögling fasse partie de ses plus zélés disciples et collaborateurs scientifiques. Premier témoin des travaux du mathématicien impérial concernant les logarithmes et leur usage dans le calcul astronomique, Mögling a fait la démonstration de sa parfaite maitrise de ces nouvelles techniques calculatoires, dans des opuscules manuscrits qui ne sont jamais sortis de la bibliothèque personnelle $\mathrm{du}$ landgrave, et dont l'impeccable sérieux scientifique n'a plus rien à voir avec les fatras théosophiques de ses premières années (voir ill. ci-contre) ${ }^{9}$. La connaissance de ces travaux permettra de corriger l'avis des éditeurs de Kepler, selon lesquels le nouveau calcul keplérien présenté dans les Tables rudolphines (1627) n'a eu aucun écho critique ni aucune réception significative en Allemagne ${ }^{10}$.

\section{_ L'invention de l'observatoire moderne, d'Uraniborg à Butzbach}

L'observatoire de Butzbach, bien que disposant d'un instrumentarium très complet et diversifié ${ }^{11}$, s'est spécialisé dans l'étude d'un phénomène détecté depuis le début de la décennie 1610 : les taches solaires. Dans une assez vaine querelle, Galilée avait revendiqué la priorité dans la découverte de ce phénomène ${ }^{12}$ qui suscite, aujourd'hui encore, l'intérêt des astrophysiciens ${ }^{13}$. Bien qu'il s'agisse d'un phénomène local, dont la nature exacte était encore mal comprise, les taches solaires constituent, en tout ce qui concerne l'astronomie et la cosmologie, le principal point d'entrée dans le débat scientifique de l'époque. Sous la houlette du landgrave, et à l'aide d'un instrument unique en son genre, Mögling a recueilli des observations sur lesquelles, peu avant sa mort, Kepler s'est appuyé pour soutenir sa dernière bataille copernicienne, propulsant de fait Butzbach au centre d'une discussion qui gagne rapidement une dimension internationale. C'est ainsi, 


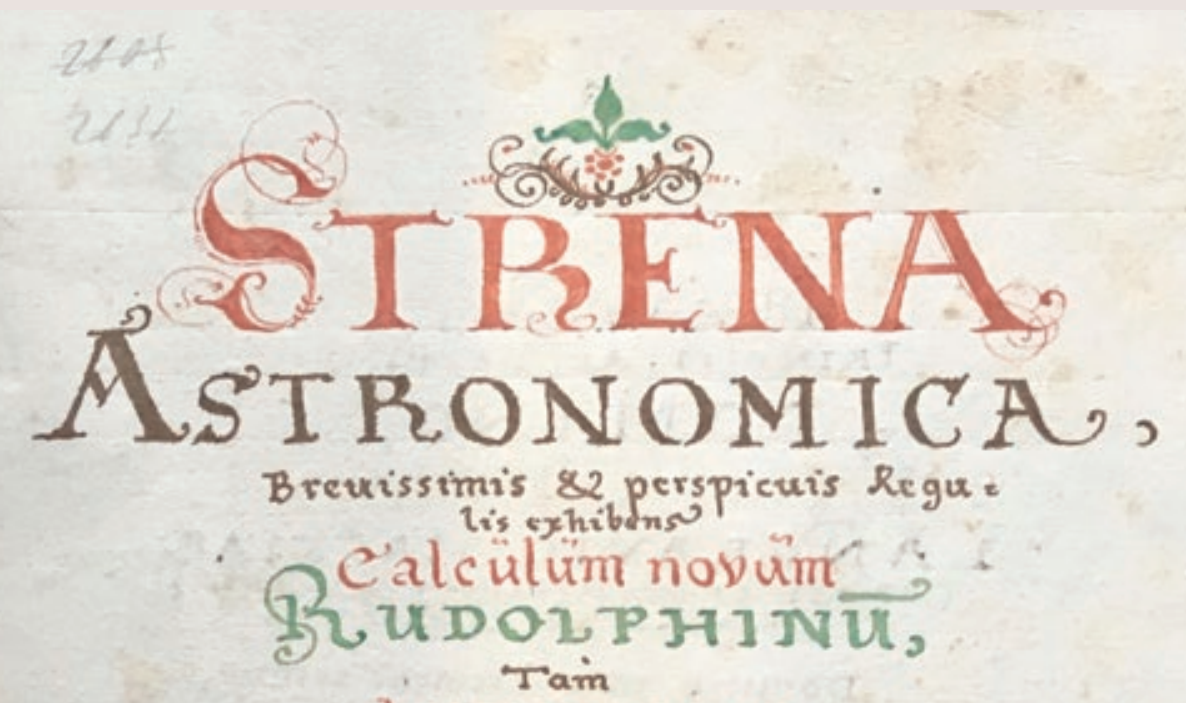

LUMINARTUM

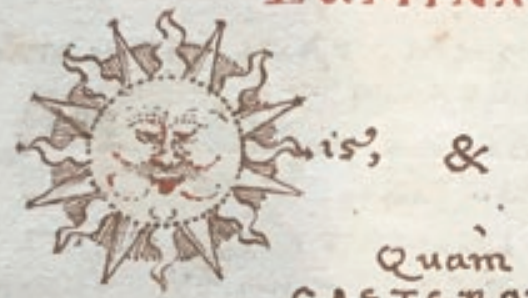

CA T\& RokUת

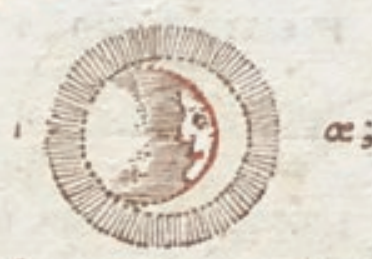

PIA $\underset{\text { supiriozum; }}{\mathrm{E}} \mathrm{I} \mathrm{ARUm}$;
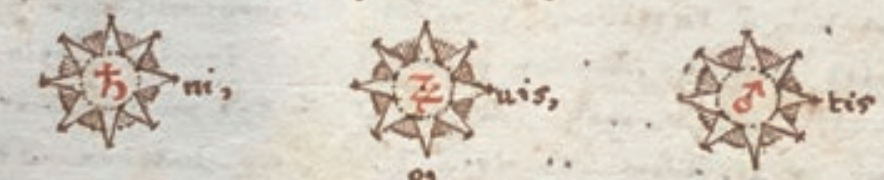

\&

Inferiorum,

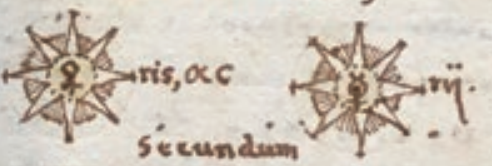

LONGUM \& 1АTUN.

Unà cüm

DE1.1 QLC11 C

COMTUTATI ONE, ex risd: Funditis,

OBSERUANDI

noDo.

M

DC, N.P11\%,

[Daniel Mögling], Strena Astronomica, Brevissimis et perspicuis Regulis exhibens Calculum novum Rudolphinum..., 1628 (coll. Universitäts- und Landesbibliothek Darmstadt [Hessisches Staatsarchiv], HS 2643) 


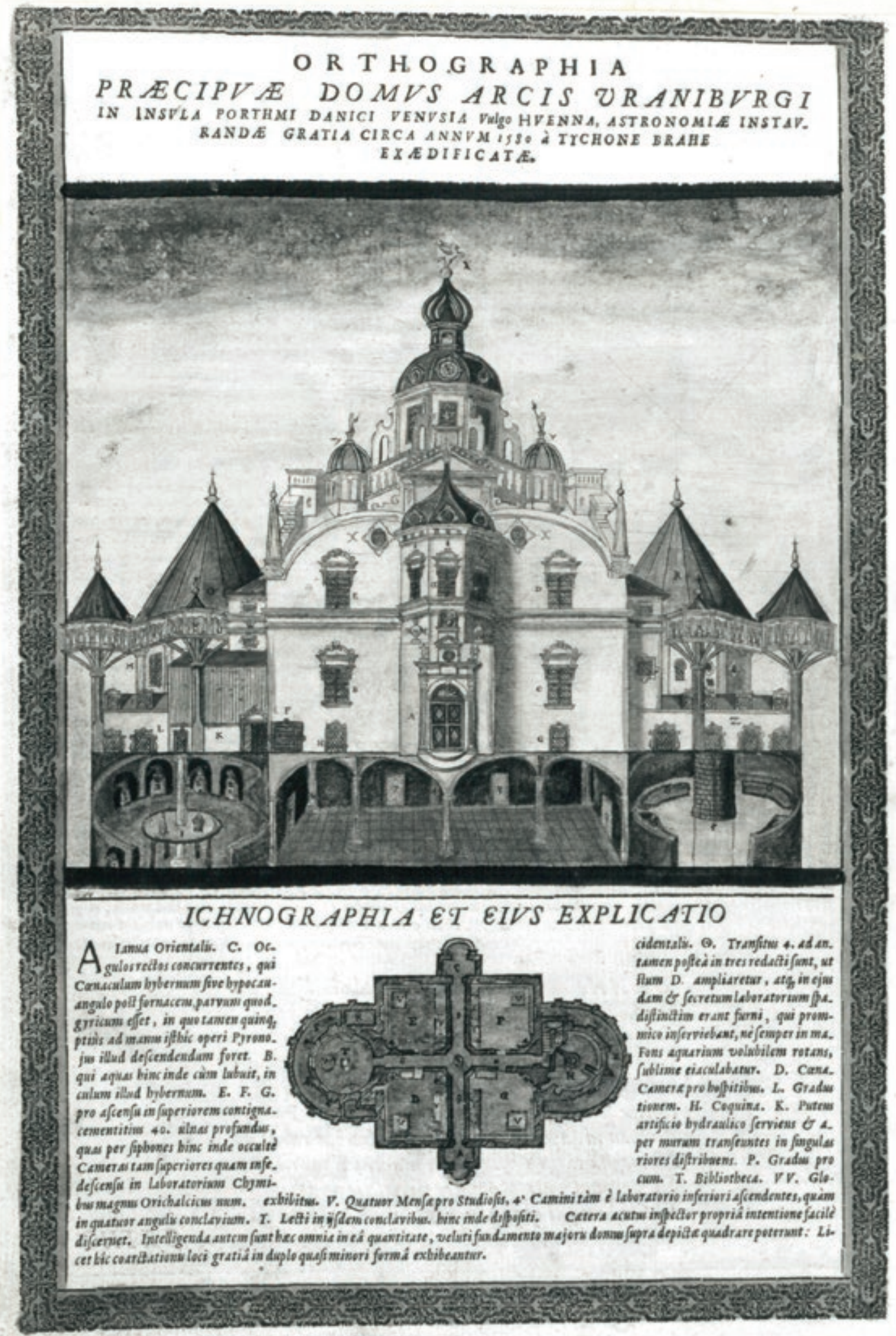

Tycho Brahe, description de l'observatoire d'Uraniborg, in Astronomiae instauratae mechanica, 1598 
par une contribution aussi discrète que précise, que Butzbach éclipse pour un temps sa rivale Kassel, pourtant réputée dans toute l'Europe savante comme le fleuron de la recherche scientifique, depuis que l'échange épistolaire de Christoph Rothmann (ca. 1550-1600), mathématicien de Kassel, avec l'astronome danois Tycho Brahe (1546-1601) avait bouleversé le champ des études astronomiques dans la seconde moitié du $16^{\mathrm{e}}$ siècle ${ }^{14}$. Unique en son genre, le conservatoire-observatoire de Butzbach n'est possible qu'en réunissant en un seul et même lieu le savoir théorique sur la construction des instruments, les moyens financiers et les compétences techniques pour leur fabrication, sans oublier l'idée, toute neuve, selon laquelle une théorie scientifique, quelle qu'elle soit, n'a de validité et même de sens qu'à condition d'être sanctionnée par une expérience décisive révélant sa nécessité ${ }^{15}$. Mais, comme l'a bien noté quelques années plus tard l'auteur du Discours de la méthode, la production de telles expériences exige du temps et des moyens qui ne sont pas à la portée d'un simple particulier ${ }^{16}$. Tycho Brahe en avait donné un célèbre (contre-) exemple, persuadant son souverain Christian IV du Danemark de construire pour lui à grands frais l'invraisemblable observatoire d'Uraniborg sur l'île de Hven (voir ill. ci-contre). L'observatoire de Butzbach en est une imitation certes pâlotte, mais surtout beaucoup plus raisonnable.

C'était là, au milieu d'un bout de désert battu par les flots, que Tycho avait installé ses instruments d'observation d'une précision révolutionnaire, ainsi qu'un moulin à papier et une imprimerie destinés à l'impression de ses ouvrages. Le bâtiment, construit selon le plan d'une architecture idéale, proposait toutes les commodités de l'époque moderne : chauffage central, eau courante, bibliothèque scientifique et espaces réservés aux chercheurs invités. Que rêver de plus ? Surgi comme par magie du génie un peu dérangé de Tycho Brahe, Uraniborg donnait un exemple, certes un peu démesuré et excessif, d'un dispositif complexe réunissant les conditions propices - et désormais indispensables - au progrès de la recherche scientifique.

Ce dispositif se trouve savamment et subtilement évoqué par le frontispice des Tabulae rudolphinae de Kepler (1627 ; voir ill. p. 69). L'histoire de ces nouvelles tables astronomiques est longue et complexe ; il suffira de retenir ici que le projet remonte à l'époque déjà ancienne où Kepler a travaillé auprès de Tycho, alors considéré, plus encore que Copernic, comme le rénovateur de l'astronomie à l'époque moderne. Pour diverses raisons, ces nouvelles Tables, tardivement parues, doivent donc célébrer la mémoire d'un savant dont Kepler ne partage aucunement les vues, et même d'un adversaire théorique qu'il était cependant obligé, pour des raisons strictement politiques, de reconnaître, à son corps défendant, comme son mentor.

L'image p. 69, d'une construction très sophistiquée, représente un tempietto aux dimensions tout à fait comparables à l'observatoire de Butzbach, au plafond duquel est représenté le système héliocentrique de Copernic ${ }^{17}$. Au centre, on voit Tycho Brahe interrogeant Copernic : « quid si sic ? " [et s'il en était ainsi ?], demande l'astronome danois en pointant l'index vers la Terre - qu'il prétend, contre Copernic, et a fortiori contre Kepler, reposer immobile au centre du monde. Ce temple pourrait être un monument à la gloire de Tycho ; mais l'image est à double fond, et le lecteur attentif comprendra vite qu'il s'agit plutôt d'un tombeau de l'astronome danois. En effet, le tempietto aux dix colonnes est surmonté par un dôme sur lequel figurent six Muses. Chacune d'elles représente les nouveaux instruments et moyens techniques de l'astronomie keplérienne : l'une des Muses tient dans une main un aimant, et dans l'autre une aiguille ; une autre tient ce qui semble être une lunette de Galilée (mais pourrait être aussi un tube optique fonctionnant comme une « camera obscura ») ; une troisième figure tient les logarithmes, que Kepler déduit d'une théorie générale des proportions ou rapports ; une autre encore tient une balance dont le soleil constitue le centre d'équilibre, et les planètes les poids - schématisant ainsi le principe fondamental d'une nouvelle «physique céleste " keplérienne dont le noble et orgueilleux Danois n'avait pas même soupçonné la possibilité. Autrement dit, le frontispice des Tabulae rudophinae montre, d'une manière assez subtile pour paraître élogieuse, la défaite de l'astronome danois incapable d'accéder aux vrais moyens d'observation et de calcul de l'astronomie moderne ! 


\section{Kepler et les taches solaires}

Le second séjour de Kepler à Butzbach a lieu en octobre 1627, immédiatement après qu'il a amené lui-même, fin septembre, quelques exemplaires des Tabulae rudolphinae à la foire de Francfort. C'est à ce moment précis qu'il prend connaissance des nouveaux instruments du landgrave, et des observations solaires menées par Daniel Mögling à l'aide d'un spectaculaire tube optique. Kepler a été le premier à publier une description de cet instrument sans exemple ${ }^{18}$ :

«Une fois ces affaires réglées, je me suis rendu à Butzbach avec le très illustre landgrave Philippe de Hesse, et j'ai pris le temps d'y contempler les instruments et exercices astronomiques du très estimé Prince ; il y aurait beaucoup à écrire pour l'éloge de ces derniers, mais ce n'est pas ici mon propos. Afin cependant de ne pas priver les lecteurs de tout l'agrément des spectacles, j'en évoquerai un seul, qui n'a pas laissé de m'émerveiller, quoique je sois particulièrement familier des observations. Dans un endroit dégagé et spacieux, on fixe un pieu d'une trentaine de pieds de hauteur ; au sommet, on place une poulie ; par cette poulie, on fait passer un câble de cabestan qui entoure un tube immense de cinquante pieds, mû à grand peine par six hommes robustes depuis son faîte ; on élève ce tube à une hauteur telle que, par son trou - qui fait la taille d'un petit pois, ou bien d'une lentille, ou même d'un grain de millet - le Soleil projette ses rayons sur une tablette blanche opposée, qui termine la cavité du tube à son extrémité. On distingue alors clairement, sur la tablette, les taches du Soleil, qui sont formées par le simple trou, sans l'interposition d'aucun verre convexe ; les figures, recueillies plusieurs jours d'affilée à midi, attestent que le mouvement des taches décrit une ligne qui est, aux solstices, perpendiculaire à la ligne méridienne, et qui forme aux équinoxes un angle complémentaire à l'obliquité de l'écliptique, en changeant d'hémisphère d'un équinoxe à l'autre [in opposito aequinoctio plagae etiam contrariae]; en sorte que cette ligne de mouvement se situe toujours dans le plan de l'écliptique. C'est pourquoi, si la tache est fixe à la surface du soleil, il s'ensuit que, de la même manière que celle-là, le globe solaire lui-même, dis-je, s'avance en suivant l'écliptique; nous en concluons ainsi qu'il tourne autour de son axe et des pôles. Ajoute cet argu- ment à tous ceux que j'ai déjà mentionnés dans l'Epit. Ast. Lib. IV p. 514 num. 4, et demande-toi si quoi que ce soit nous empêche encore de tenir pour très certaine la proposition suivante : LE SOLEIL EST L'ORIGINE DU MOUVEMENT DES PLANÈTES ».

Mögling n'ayant jamais rien publié sur le sujet, les lecteurs devaient croire Kepler sur parole. Or les observations de Butzbach, tout récemment tirées de l'oubli où elles sommeillent depuis bientôt quatre cents ans, confirment entièrement les propos de l'astronome impérial - à un détail près qui sera bientôt corrigé par l'astronome Wilhelm Schickard ${ }^{19}$. Le soin et l'exactitude apportés par Mögling à l'observation des taches solaires fait du manuscrit HS 228 (voir ill. p. 71) un document d'un intérêt inestimable pour l'histoire des sciences : non seulement comme témoignage de première importance sur la discipline émergente qu'est la physique solaire, mais aussi, voire davantage encore, parce que la défense par Kepler de la valeur de ces observations menées de manière quasi confidentielle a déclenché, venant de l'étranger (Philipp Lansbergen, Martin Hortensius, Isaac Beeckman) une vague d'hostilité sans précédent, et une mise en cause des fondements mathématiques, optiques et observationnels de sa théorie. Cette attaque coordonnée vient des Provinces-Unies, et plus spécialement de Middelburg, capitale de la province de Zélande et, pourrait-on dire, capitale du télescope, qu'Hortensius et Beeckman préfèrent au tube optique pour l'observation des taches solaires. Or la discussion théorique qui oppose les Zélandais à Kepler sur les avantages respectifs du télescope à lentille et du simple tube optique s'avère, en dépit de son apparence modeste et localisée, d'une très grande importance pour l'histoire de la science classique, puisque c'est des brumes de cette discussion qu'émergera bientôt, comme un soleil matinal, la Dioptrique de Descartes.

\section{NOTES}

1- Correspondance inédite de Henri IV, roi de France et de Navarre, avec Maurice-le-Savant, landgrave de Hesse, par M. de Rommel, Paris, Renouard, 1840

2- Selon l'adresse d'une lettre non datée, dont la minute est conservée au Hessisches Staatsarchiv [Universitäts- und Landesbibliothek Darmstadt]. Nous remercions Susan Kleine et Birgit Reeg-Lumma pour l'aide qu'elles nous ont apportée dans l'exploration du fonds manuscrit de Darmstadt. 


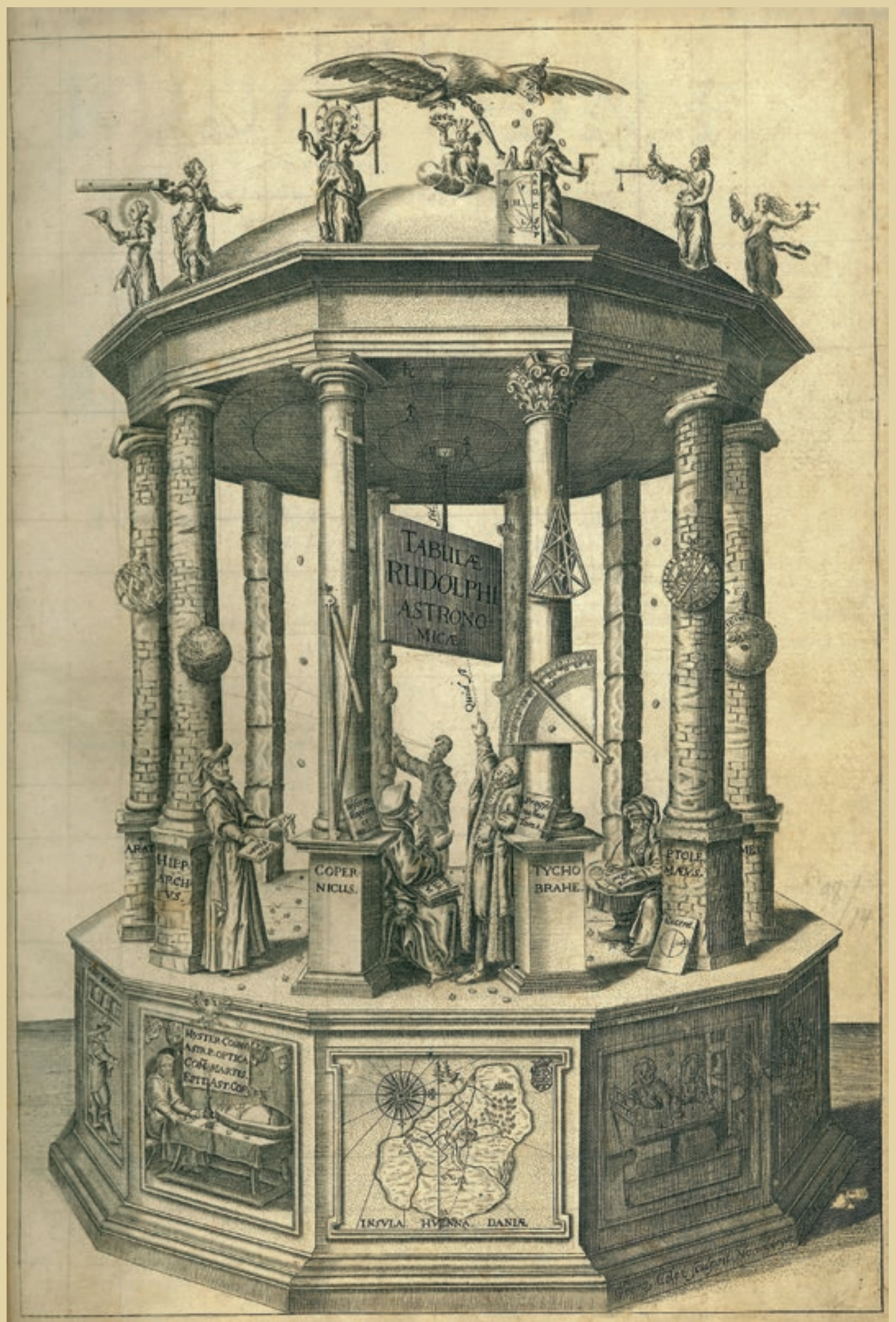

J. Kepler, frontispice des Tabulae ruldophinae (1627), in Michael Raggsted : " About the cover: Kepler and the Rudolphine Tables ", in Bulletin (New series) of the American Mathematical Society, vol. $50, n^{\circ} 4$, octobre 2013 , p. 629-639 
3- Sur la diffusion du télescope, voir Massimo Bucciantini, Michele Camerota, Franco Giudice : Galileo's Telescope. A european Story, Cambridge (Massachussetts), London, Harvard University Press, 2015. Sur le Messager céleste (Sidereus nuncius) de Galilée (1610), voir l'édition d'Isabelle Pantin (Paris, Les Belles Lettres, 1992), et Philippe Hamou, La mutation du visible. Essai sur la portée épistémologique des instruments d'optique au XVII siècle (vol. 1), Lille, Presses universitaires du Septentrion, 1999.

4- Inventar über die Reißkammer Landgrav Philipps von Butzbach, geschrieben von Daniel Mögling im November 1628 mit Nachträgen von diesem und anderen bis in die vierziger Jahre. Cet inventaire est disponible en version numérisée sur le site de l'Universitäts- und Landesbibliothek Darmstadt : http://tudigit.ulb.tu-darmstadt.de/ show/Hs-3020. L'inventaire mentionne aussi plusieurs instruments de musique, Mögling ayant travaillé à la réalisation d'un automate musical - un clavecin jouant de la musique tout seul dès l'apparition $\mathrm{du}$ soleil. Le présent travail se fonde également sur le dépouillement de deux codices, astronomique et astrologique, constitués par Daniel Mögling : HS 2608 et HS 2526. Le HS 2608 comporte notamment un bref manuscrit astrologique de Kepler, édité dans le dernier volume de ses Gesammelte Werke (XXI, 2.2, München, Beck, 2009, bearbeitet von Friederike Boockmann und Daniel A. Di Liscia). Malheureusement, une faute de plume dans le report d'une date a fait que le manuscrit keplérien a été mal contextualisé : il n'est pas arrivé à Butzbach en 1612, comme l'affirme KGW XXI, 2.2, p. 507 et 633 , mais en 1621 , lorsque Kepler a fait son premier séjour à Butzbach. Les éditeurs n'ont par ailleurs pas noté que l'exemple traité dans ce texte est un horoscope pour une naissance datée du 25[26].12.1581, i. e. la date de naissance du landgrave Philippe. Il s'agit donc selon toute vraisemblance d'un texte offert par Kepler au landgrave lors de son premier séjour en 1621.

5- L'expression peut paraître excessive, mais elle est, avec Kepler, à prendre au pied de la lettre, lui-même se définissant comme « sacerdos libri naturae » (voir par ex. Kepler à Georg Herwart von Hohenburg, 26.3.1598, lettre n $91, \mathrm{KGW} 13$, 193). Sur ce phénomène moderne de sécularisation, voir les indications suggestives de David Aubin : « Modèles de scientificité dans les observatoires : les églises de l'âge positiviste ? ", in Revue des sciences religieuses, 87, $\mathrm{n}^{\circ} 4$ (2013), p. 451-461.

6- Ce terme, qu'on ne trouve dans aucun dictionnaire, mais qui apparaît dans certains inventaires dont celui de la collection d'art (Kunstkammer) de la cour de Dresde en 1587, semble venir d'un des sens du verbe « reissen » (dessiner). Il désignerait ainsi le lieu où est stocké le matériel de dessin et de mesure destiné aux travaux scientifiques. Qu'une bibliothèque, ainsi que des instruments divers, lui soient adjoints semble naturel pour la constitution de ce qu'on peut qualifier de « dispositif de savoir ».

7- Voir la notice de la Deutsche Biographie établie par Ulrich Neumann (https://www.deutsche-biographie.de/sfz63848.html), qui ne donne d'ailleurs pas une liste bibliographique complète des travaux de Mögling. La plus importante source documentaire sur l'activité scientifique de Mögling entre 1621 et 1632 vient de l'édition de sa correspondance avec l'astronome et orientaliste Wilhelm Schickard. Voir W. Schickard, Briefwechsel, 1616-1632, éd. Friedrich Seck, Stuttgart-Bad Cannstatt, Frommann-Holzboog, 2002 (2 Bände).

8- Sur cet épisode, voir Ivo Schneider, Johannes Faulhaber, 15801635. Rechenmeister in einer Welt des Umbruchs, Basel, Boston, Berlin, Birkhaüser, 1993 ; Kurt Hawlitschek : Johann Faulhaber, 1580-1635. Eine Blütezeit der mathematischen Wissenschaften in Ulm, Ulm, Veröffentlichungen der Stadtbibliothek, 1995. Selon une hypothèse déjà ancienne, le jeune Descartes aurait pu assister à ce procès : voir Lüder Gäbe, Descartes Selbstkritik. Untersuchungen zur Philosophie des jungen Descartes, Hamburg, Meiner, 1972. Cette hypothèse a servi de fil conducteur à la thèse dont les Presses universitaires de Strasbourg publient aujourd'hui une édition revue et augmentée : Édouard Mehl, Descartes en Allemagne. Le contexte allemand de l'élaboration de la science cartésienne, Strasbourg, Presses universitaires de Strasbourg, [2001], 2019².

9- C'est par l'entremise et à la demande du landgrave Philippe III qu'a été publié le traité de Kepler sur les logarithmes : Chilias logarithmorum ad totidem numeros rotundos... ad Illustriß. Principem et Dominum, Dn. Philippum Landgravium Hassiae, \&c., Marburg, Caspar Chemlin, 1624.

10- KGW 10, Nachbericht (Franz Hammer), 85*

11- L'inventaire (environ 180 entrées) mentionne plusieurs globes célestes, quadrants, sextants, compas géométriques, lunette de Galilée, cristaux pour étudier la diffraction et réfraction de la lumière, boussoles et instruments magnétiques pour l'orientation et la mesure de la déclinaison magnétique... La plupart de ces instruments sont construits en laiton et non plus en bois, ce qui augmente considérablement leur précision, en même temps que leur coût de fabrication. Voir Ernst Zinner, Deutsche und Niederländische astronomische Instrumente des 11.-18. Jahrhunderts, München, Beck, 1965. L'unique description du grand globe céleste construit par le landgrave entre 1621 et 1632 se trouve dans une des premières sommes de muséologie répertoriant les cabinets de curiosités au $17^{e}$ siècle, due à un érudit de Gießen, Michael Bernhard Valentini (1657-1729) : Museum Museorum... [zweiter Teil], Frankfurt am Main, 1714, appendix XVI, p. 57. On apprend dans ce seul et unique document que le globe représentait le portrait du landgrave In corde Leonis (= Régulus, $\alpha$ Leonis) et de son épouse au lieu de Spica Virginis ( $\alpha$ Virginis) - ces deux étoiles formant avec Arcturus ( $\alpha$ Bootis) un astérisme traditionnellement désigné comme le «triangle de printemps ».

12- La dispute pour la primeur de cette découverte marque le début des hostilités entre Galilée et les jésuites, représentés par le père Christoph Scheiner (1575-1650). En réalité, les taches ont été observées de manière indépendante par David Fabricius : voir M. Folkerts, « Johannes Kepler und David Fabricius », in Kepler. La physique céleste. Autour de l'Astronomia nova (1609), éd. Édouard Mehl, Paris, Les Belles Lettres, 2011, p. 43-66.

13- Comme en témoigne l'envoi par la NASA de la sonde Parker (Parker Solar Probe) en août 2018. Cette sonde a pour mission d'observer au plus près l'atmosphère et la météorologie solaires. À l'époque qui nous occupe, la nature physique des taches solaires et la composition de l'atmosphère qui enveloppe le soleil ne sont pas encore connus, mais c'est justement Kepler qui a déduit, sur la base des observations de Butzbach, l'existence d'un milieu circumsolaire aux propriétés physiques et optiques spécifiques.

14- J. Bernoulli cite une lettre inédite de Willebrord Snell au landgrave Moritz von Hessen-Kassel, exhortant celui-ci à remettre en route le programme des observations délaissé depuis la succession de son père Guillaume IV; cf. Jean Bernoulli, Lettres astronomiques où l'on donne une idée de l'état actuel de l'astronomie pratique dans plusieurs villes de l'Europe (1771, p. 49). Sur l'activité astronomique à Kassel autour de Guillaume IV et du copernicien Christoph Rothmann, voir Jürgen Hamel, Die astronomischen Forschungen in Kassel unter Wilhelm IV (Acta Historica Astronomiae, vol. 2), Harri Deutsch Verlag, 2002 ; Christoph Rothmann's Discourse on the Comet of 1585. An Edition and Translation, with Accompanying Essays, Miguel Á. Granada, Adam Mosley \& Nicholas Jardine (éd.), Leiden-Boston, Brill, 2014.

15- Voir à ce sujet l'éloquente lettre du landgrave Philipp à Kepler, datée du mois de juin 1623 (lettre $n^{\circ}$ 954, KGW 18, 130) : les hypothèses de l'astronomie reposent sur deux pieds, à savoir les observations 

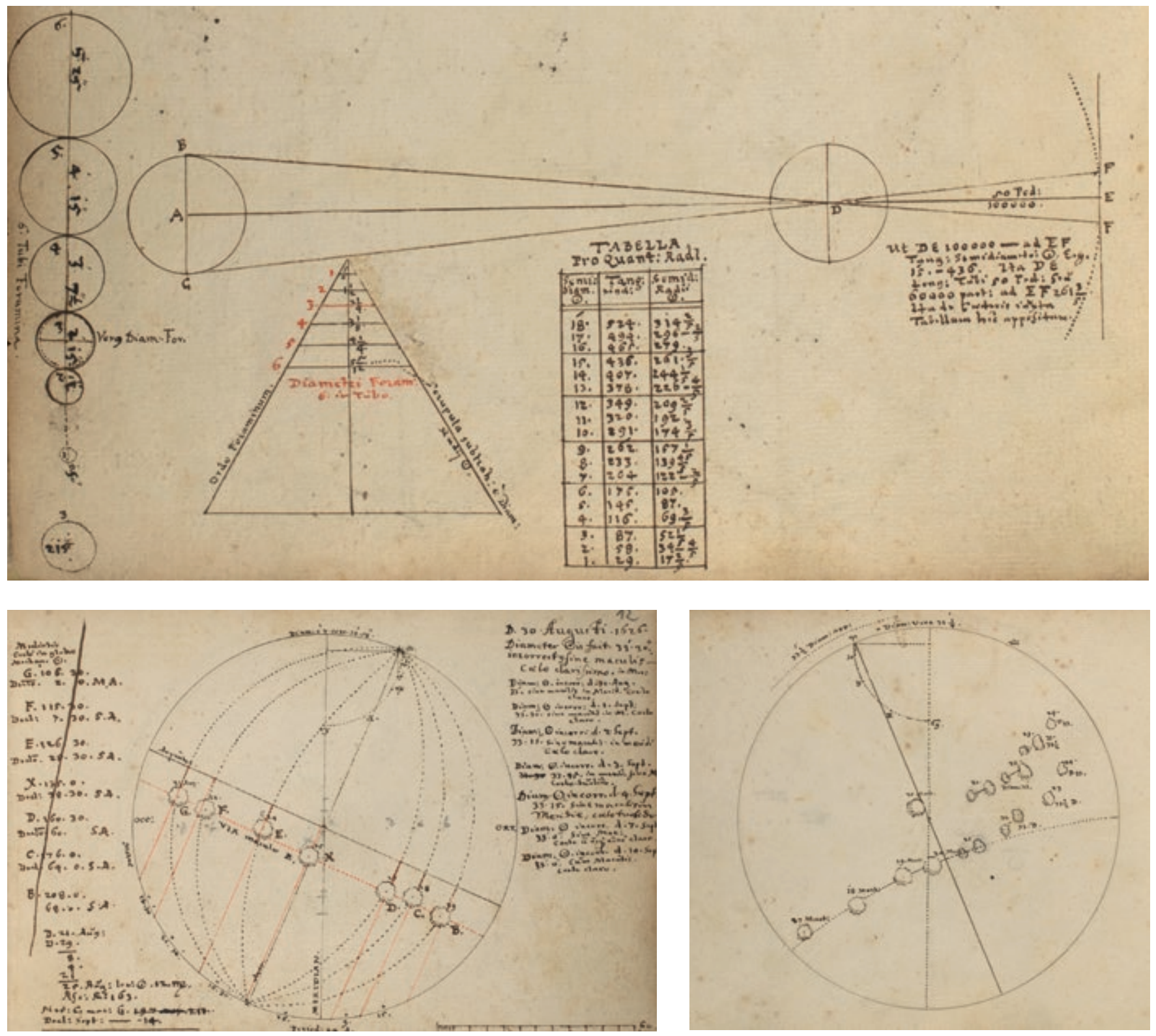

[Daniel Mögling], Observationes macularum solarium, 1626-1630

(coll. Universitäts- und Landesbibliothek Darmstadt, HS 228, ff. $2 v^{\circ} ; 12 \mathrm{r}^{\circ} ; 36 \mathrm{r}^{\circ}$ )

et le calcul. Pour les premières, il faut des instruments, mais le landgrave évoque la difficulté de les utiliser avec la plus grande exactitude, sans que ne s'y glisse le risque d'erreurs. Pour le second, il faut des méthodes de calcul, et c'est pour cette raison que le landgrave demande à Kepler de lui fournir des tables logarithmiques destinées à abréger des calculs autrement interminables.

16- R. Descartes, Discours de la méthode pour bien conduire sa raison et trouver la vérité dans les sciences (1637), in Euvres complètes sous la direction de Jean-Marie Beyssade et Denis Kambouchner, Paris, Gallimard (TEL), 2009, p. 130 : «De sorte que, s'il y avait au monde quelqu'un que l'on sût assurément être capable de trouver les plus grandes choses et les plus utiles au public qui puissent être, et que pour cette cause les autres hommes s'efforçassent par tous les moyens de l'aider à venir à bout de ses desseins, je ne vois pas qu'ils pussent autre chose pour lui, sinon fournir aux frais des expériences dont il aurait besoin, et du reste empêcher que son loisir ne lui fût ôté par l'importunité de personne ».
17- Sur le frontispice des Tabulae rudolphinae, voir Michael Rågsted : "About the cover: Kepler and the Rudolphine Tables ", in Bulletin (New series) of the American Mathematical Society, vol. 50, $\mathrm{n}^{\circ} 4$, octobre 2013, p. 629-639.

18- Kepler, Ad Epistolam.... Jacobi Bartschii... Responsio : de computatione et editione Ephemeridum, KGW 11, 1, 469, 1. 18-42

19- Dans sa Pars responsi ad epistolas P. Gassendi [...] de Mercurio sub sole viso et aliis novitatibus uranicis..., Tübingen, Brunnius, 1632, Wilhelm Schickard nomme Daniel Mögling (ce que Kepler ne fait pas) et corrige la description de Kepler en ce qui concerne le diamètre de l'ouverture, ou «foramen ", par lequel la lumière entre dans le tube : il n'est pas de la taille d'un grain de mil, comme le dit Kepler - qui n'a probablement pas pu s'en approcher assez pour le constater de visu -, mais d'environ $1,5 \mathrm{~cm}$, soit la taille d'une olive. En réalité, comme on le voit ci-dessus, le tube dispose de six diamètres d'ouverture dont la taille varie de $1 / 2$ pouce à $3,5 \mathrm{~cm}$. Kepler a en fait décrit la taille approximative des trois plus petits (« de la taille d'un petit pois, d'une lentille, ou même d'un grain de millet »). 